O16 (continued)

Study Design, Setting, Participants, and Intervention: The Healthy Outcomes for Teens (HOT) Project is an interactive website for middle-school students with 7 modules, including 6 videos for observational learning. A duplicate site was created replacing videos with narratives. Students $(\mathrm{n}=41)$, recruited from 6th-8th grade Physical Education classes, were divided into 2 groups, video-first and narration-first. Students viewed one site first, and then switched to view the other site in a cross-over design. Focus groups followed each website viewing by one week and were recorded, transcribed, and analyzed by thematic analysis.

Outcome, Measures and Analysis: Recall and comprehension of 6 videos and 6 narratives.

Results: The video-first group provided more details of the scenarios, and more accurately comprehended the intended messages compared to the narrative-first group. Students felt the scenarios were believable and should remain on the website. A small group of students agreed that the choices modeled needed to be more challenging, while another small fraction agreed the scenarios should be longer. Preference for watching videos did not differ by gender; however, gender may have influenced which scenarios were most remembered by boys and girls.

Conclusions and Implications: Embedded videos could augment the educational process and could be a way to improve long-term retention of nutrition information in adolescents.

Funding: Kraft Foods, University of Illinois, Division of Nutritional Sciences

\section{Impact Assessment of a Behavior Change Communication Program in Niger}

Naoko Horii, MPH, MA, Naoko.Horii@gmail.com, Centre Population \& D, 19 rue de Jacob, Paris, 75006, France; O. Habi, MS, Institut National de Statistique (INS) Niger; A. Dangana, MS; A. Maina, MS; S. Alzouma, MS

Objectives: This study assesses the impact of behavior change communication on early initiation of breastfeeding in Niger.

Study Design: A secondary analysis of a post intervention survey conducted in Niger in 2011. The study population includes 2,091 women aged 15-49 years with children less than 23 months. They are categorized into an intervention group exposed to the communication program and control groups not exposed to the intervention.

Outcome, Measures and Analysis: The outcome variable is early initiation of breastfeeding, which is defined as putting a child to breast within 1 hour of birth. Independent variables include different types of communication program activities. Other variables include socio-demographic, economic, environmental and health seeking behavior related indicators.

Chi-square tests identify the determinants of early initiation of breastfeeding based on the entire population of the study sample. Stratified bivariate analysis and multivariate logistic regression examine the influence of the communication program on initiation of breastfeeding.
Results: Based on the entire population, home visits by trained community volunteers to mothers showed slight but positive influence on improved initiation of breastfeeding which increased by $15 \%$ compared to mothers who have not received home visits (95\%CI: -11, 34). Regardless of socio-economic vulnerability, mothers' involvement in breastfeeding promotion increased by $99 \%$ their early initiation of breastfeeding compared to those not involved in any health promotion activities (95\%CI: 39, 185).

Conclusions: The study suggests that a community based participatory approach could be an equity-focused model for behavior change in early initiation of breastfeeding.

Funding: None

\section{Food and Beverage Dietary Guidelines and Soy Gardening Training in Low-Income Households in Qwa-Qwa, South Africa}

AbdulkadirEgal,PhD, abdul@vut.ac.za, Vaal University of Technology, Private Bag X021, Vanderbijlpark, 1910, South Africa; $W$. Oldewage-Theron, $P h D, R D$

Objective: To measure the knowledge of the South African food-based dietary guidelines (FBDG), as well as of soy gardening and uses of soy, before and after a nutrition education training programme (NEP).

Study Design, Setting, Participants, and Intervention: The four phases of the Food and Agriculture Organization of the United Nations framework for nutrition education were used as a basis for the study. A structured nutrition education questionnaire (NEQ) consisting of 32 questions was developed. The NEQ was used to collect data both at baseline and at the end of a NEP in 75 purposively selected households. Descriptive statistics (frequencies) and paired t-tests were done to determine the statistically significant $(\mathrm{p}<0.05)$ differences in knowledge before and after the NEP.

Outcome, Measures and Analysis: Pre and post training of both FDGB and soy gardening where measured using SPSS for significant difference.

Results: At baseline, the results showed a good knowledge of the FBDG as the majority of the respondents (66\%) answered the questions correctly. Regarding soy, the knowledge of respondents improved significantly $(\mathrm{p}<0.05)$ from $65.0 \%$ correctly answered questions before the NEP to $74.1 \%$ after the NEP.

Conclusions and Implications: The NEP proved to be successful in teaching the caregivers about soy gardening and soy use in the household.

Funding: South African-Netherlands Research Programme on Alternatives in Development

\section{Eat Smart, Live Strong Intervention Increases Fruit and Vegetable Consumption among Low-Income Older Adults}

Sheryl Cates, BS, scc@rti.org, RTI International, 3040 Conrwallis Road, Research Triangle Park, NC 27709; O. J. Santiago, PhD, Michigan State University Extension; J. Hersey, PhD, RTI International; J. Blitstein, PhD; K. Kosa, MS; A. Singh, PhD, USDA, FNS; D. Berman, PhD

Continued on page $S 104$ 